\title{
Loss of porin function in dopaminergic neurons of Drosophila is suppressed by Buffy
}

P. Githure M'Angale and Brian E. Staveley*

\begin{abstract}
Background: Mitochondrial porin, also known as the voltage-dependent anion channel (VDAC), is a multi-functional channel protein that shuttles metabolites between the mitochondria and the cytosol and implicated in cellular life and death decisions. The inhibition of porin under the control of neuronal Ddc-Gal4 result in short lifespan and in an age-dependent loss in locomotor function, phenotypes that are strongly associated with Drosophila models of Parkinson disease.
\end{abstract}

Methods: Loss of porin function was achieved through exploitation of RNA interference while derivative lines were generated by homologous recombination and tested by PCR. The UAS/Gal4 expression system was exploited with directed expression in neurons achieved with the use of the Dopa decarboxylase and in the developing eye with the Glass multiple reporter transgenes. Statistical analyses for ageing assay employed Log rank (Mantel-Cox) test, climbing indices were fitted with a non-linear curve and confidence intervals compared at 95\%. Biometric analysis of the eye phenotypes was obtained by unpaired student $T$-test.

Results: The expression of $a$-synuclein in neuronal populations that include dopamine producing neurons under the control of Ddc-Gal4 produces a robust Parkinson disease model, and results in severely reduced lifespan and locomotor dysfunction. In addition, the porin-induced phenotypes are greatly suppressed when the pro-survival BCl-2 homologue Buffy is overexpressed in these neurons and in the developing eye adding to the cellular advantages of altered expression of this anti-apoptotic gene. When we co-expressed $a$-synuclein along with porin, it results in a decrease in lifespan and impaired climbing ability. This enhancement of the $a$-synuclein-induced phenotypes observed in neurons was demonstrated in the neuron rich eye, where the simultaneous co-expression of porin-RNAi and a-synuclein resulted in an enhanced eye phenotype, marked by reduced number of ommatidia and increased disarray of the ommatidia.

Conclusions: The inhibition of porin in dopaminergic neurons among others result in reduced lifespan and agedependent loss in climbing ability, phenotypes that are suppressed by the overexpression of the sole pro-survival $B c 1-2$ homologue Buffy. The inhibition of porin phenocopies Parkinson disease phenotypes in Drosophila, while the overexpression of Buffy can counteract these phenotypes to improve the overall "healthspan" of the organism.

Keywords: a-synuclein, Buffy, Dopaminergic neurons, Mitochondria, Porin, Parkinson disease

\footnotetext{
* Correspondence: bestave@mun.ca

Department of Biology, Memorial University of Newfoundland, St. John's,

Newfoundland \& Labrador A1B 3X9, Canada
}

(c) The Author(s). 2016 Open Access This article is distributed under the terms of the Creative Commons Attribution 4.0 International License (http://creativecommons.org/licenses/by/4.0/), which permits unrestricted use, distribution, and reproduction in any medium, provided you give appropriate credit to the original author(s) and the source, provide a link to the Creative Commons license, and indicate if changes were made. The Creative Commons Public Domain Dedication waiver (http://creativecommons.org/publicdomain/zero/1.0/) applies to the data made available in this article, unless otherwise stated. 


\section{Background}

The voltage-dependent anion channel (VDAC), also known as mitochondrial porin, consists of small poreforming proteins present in the outer mitochondrial membrane that act to shuttle nucleotides, metabolites and ions between the mitochondria and the cytoplasm $[1,2]$. Porin is a multi-functional protein and is involved in the regulation of metabolism and energetic functions of the mitochondria and a constituent of the mitochondrial permeability transition pore (PTP) [3]. Porin is involved in apoptosis, metabolite transport, calcium transport and signalling, ATP transport, reactive oxygen species transport and endoplasmic reticulum mitochondrial crosstalk [3-5]. As thus porin appears to be a convergence point for cell death and survival signals, mediated by its association with a variety of ligands and proteins. Porin is implicated in mitochondria-mediated apoptosis and in regulation of apoptosis through interaction with pro-survival proteins [3]. It interacts with the pro-survival hexokinase to mediate its anti-apoptotic activity [3,6], and the $\mathrm{Bcl}-2$ family of proteins to regulate mitochondria-mediated apoptosis $[7,8]$. This association can induce cell survival or death.

The porin gene is associated with several neurodegenerative disorders including Alzheimer disease [9], Down syndrome [10], and dopamine-induced apoptosis [11]. The association of porin with Parkinson disease-associated gene products has been established, where it recruits parkin to defective mitochondria to promote mitophagy [12], and shows high affinity interaction with $\alpha$-synuclein to regulate mitochondrial-induced toxicity [13]. This study suggests that $\alpha$-synuclein translocate to the mitochondria via porin to target complexes of the mitochondrial respiratory chain. The accumulation and aggregation of abnormal $\alpha$-synuclein was shown to down-regulate porin [14] and possibly regulate mitochondrial permeability [15]. The association between the PD gene $\alpha$-synuclein and the mitochondrial channel porin appears to be important in the progression of PD. The initial Drosophila PD model employed the expression of human $\alpha$-synuclein transgene to generate the PD-like phenotypes [16], that are commonly known as the $\alpha$-synuclein-induced phenotypes. The success of this model anchors on its ability to phenocopy features of human PD such as the age-dependent loss in locomotor function and therefore, has found application in the study of $\alpha$-synuclein-induced degeneration [16-23]. The use of the bipartite UAS/GAL4 expression system [24], and the remarkable number of promoters or enhancers available, of which TH-Gal4, elav-Gal4 and Ddc-Gal4 are utilized in modelling PD in flies [16-23], makes Drosophila a useful and albeit a powerful model organism.

The loss of function of Drosophila porin/VDAC has been shown to result in mitochondrial morphological defects $[25,26]$. These mitochondrial defects were accompanied by locomotor dysfunction and male sterility. In addition, porin mutants displayed neurological and muscular defects, mitochondrial respiratory defects, and abnormalities in synaptic transmission and mitochondrial distribution in motor neurons. Here we suppressed porin by RNA interference in Drosophila neurons under the control of the dopa decarboxylase transgene and analysed longevity and locomotor ability. Further we co-expressed porin-RNAi with $\alpha$-synuclein to investigate its effects in the well-studied Drosophila PD model. The association of porin with Bcl-2 members is well documented, we have demonstrated the benefits of overexpression of the sole anti-apoptotic $B c l-2$ member Buffy in conditions of stress $[27,28]$, as thus, we overexpressed Buffy along with porin-RNAi. In addition, we altered the expression of porin in the Drosophila developing eye and co-expressed with $\alpha$-synuclein and Buffy.

\section{Methods \\ Bioinformatic analysis}

The protein sequences were sourced from National Center for Biotechnology Information (NCBI; http:// www.ncbi.nlm.nih.gov/protein/) while conserved domains were identified using the NCBI Conserved Domain Database (CDD; http://www.ncbi.nlm.nih.gov/cdd) [29] and the Eukaryotic Linear Motif [30] (http://elm.eu.org/) which focuses on annotation and detection of eukaryotic linear motifs (ELMs) or short linear motifs (SLiMs). Clustal Omega multiple sequence alignment (http:// www.ebi.ac.uk/Tools/msa/clustalo/) [31, 32] was used to show conservation of the porin3_VDAC domain in the selected organisms. The nuclear export signal (NES) was predicted by NetNES (http://www.cbs.dtu.dk/services/ NetNES/) [33] and TMpred, a program that predicts membrane-spanning regions and their orientation. The algorithm is based on the statistical analysis of TMbase, a database of naturally occurring transmembrane proteins (http://www.ch.embnet.org/software/TMPRED_form.html).

\section{Drosophila media and culture}

Stocks and crosses were maintained on standard cornmeal/molasses/yeast/agar media treated with propionic acid and methylparaben to inhibit fungal growth. Stocks were maintained on solid media for 2 to 3 weeks before transfer onto new media to re-culture. Stocks were kept at room temperature $\left(22^{\circ} \mathrm{C} \pm 2{ }^{\circ} \mathrm{C}\right)$ while crosses and experiments were carried out at 25 and $29^{\circ} \mathrm{C}$.

\section{Drosophila stocks}

The $P\{K K 107645\} V I E-260 B$ hereby referred to as UAS-porinRNAi (1) was obtained from Vienna Drosophila Resource Center, $y[1] \quad v[1] ; \quad P\{y[+t 7.7] \quad v[+t 1.8]=$ TRiP.JF03251 $\} a t t P 2 /$ $T M 3, S b[1]$ hereby known as UAS-porin-RNAi (2). Porin 
expression patterns are detailed in FlyBase http://flybase.org/ reports/FBgn0004363.html, and in the Berkeley Drosophila Genome Project (BDGP; http://flybase.org/reports/ FBgn0004363.html) [34]. Similarly, a thorough expression study was performed by Olivia et al., 2002 and showed a wide range of patterns [35]. GMR-Gal4 [36] and UAS-lacZ flies were obtained from the Bloomington Drosophila Stock Center at Indiana University. UAS- $\alpha$-synuclein [16] was generously provided by Dr. M. Feany of Harvard Medical School, Ddc-Gal4 [37] by Dr. J. Hirsch of University of Virginia and UAS-Buffy [38] by Dr. L. Quinn of University of Melbourne. Studies to establish the expression pattern of Buffy have previously been performed [38, 39]. They detected Buffy mRNA via RT-PCR at all developmental stages, with the strongest expression being at the late larval/ early pupal stage [38]. The expression patterns correlate with regions of cell death and occurs in the same pattern as the procell death $\operatorname{Debcl}[38,40]$. Additional expression data is found on FlyBase http://flybase.org/reports/FBgn0040491.html.

\section{Drosophila derivative lines}

The UAS- $\alpha$-synuclein/CyO; Ddc-Gal4/TM3, UAS- $\alpha$ synuclein/CyO; GMR-Gal4, UAS-Buffy/CyO; Ddc-Gal4 and $U A S-B u f f y / C y O ; G M R-G a l 4$ derivative lines were generated using standard homologous recombination methods that we have previously described [41, 42] and were used for the overexpression of either $\alpha$-synuclein or Buffy in DA and other neurons using the Ddc-Gal4 transgene or in the developing eye using the GMR-Gal4 transgene. PCR reaction was used to determine the amplification of DNA products and Gel electrophoresis was used for confirmation of recombination events via presence of the PCR product.

\section{Ageing assay}

The analysis for survival was performed following a protocol that has previously been described [27, 43]. But briefly, from each genotype crosses were made and a cohort of at least two hundred flies collected and aged. Flies were considered dead when they did not display movement upon agitation [44]. Survival curves were compared using the log-rank (Mantel-Cox) test and significance was determined at $95 \%$, at a $P$-value less than or equal to 0.05 with Bonferroni correction.

\section{Climbing assay}

Analysis for climbing ability was determined using a standard protocol that was described in our laboratory [45]. This assay scores the flies ability to climb over their lifetime and analyses 50 males from every genotype. Climbing indices obtained were analysed using GraphPad Prism version 5.04 and climbing curves were fitted using non-linear regression. Comparisons were done at a 95\% confidence interval with a $P$-value threshold of less than 0.05 considered significant.

\section{Scanning electron microscopy of the Drosophila eye}

The Drosophila eyes for scanning electron microscopy and analysis were prepared following a standard protocol, as previously described [27]. At least 10 different eye images per genotype were analysed using the National Institutes of Health (NIH) ImageJ software [46]. The proportion of the disrupted eye area was calculated as detailed in a previous publication [47]. Statistical comparisons were evaluated using a one-way analysis of variance followed by a Dunnett's multiple comparison tests. $P$-values less than 0.05 were considered significant.

\section{Results}

The human and Drosophila porin domain is conserved There is $62 \%$ identity and $77 \%$ similarity between the human porin (VDAC) and the Drosophila melanogaster porin protein sequences, with very high conservation within the Porin3_VDAC domain (Fig. 1). The putative dimerization interface and putative determinants of voltage-gated binding sites are well conserved as determined by an NCBI conserved domain search [29]. A Eukaryotic linear motif (ELM) resource search for functional sites [48] in the Drosophila transcript indicates the presence of an inhibitor of apoptosis binding motif (IBM) that function in the abrogation of caspase inhibition by inhibitors of apoptosis (IAPs) at amino acids 1 to 5, an Atg8 binding motif at amino acids 5 to 9, a nuclear export signal (NES) at amino acids 91 to 98 , a PDZ domain at amino acids 277 to 282 and a transmembrane domain predicted by TMpred.

Inhibition of porin in neurons decreases lifespan and severely impairs locomotor function, phenotypes that are suppressed by Buffy overexpression

The expression of porin-RNAi in Ddc-Gal4-expressing neurons results in a slightly decreased lifespan and severely impaired locomotor function as shown by the two RNAi lines that we tested. The median lifespan for these flies was 48 and 52 days when compared to 68 days for the controls as determined by Log-rank (Mantel-Cox) test with a $p<0.0001$ (Fig. 2a). When porin is suppressed in these neurons, the flies have impaired locomotor ability as determined by comparison of $\mathrm{CI}$ after the nonlinear fit of the climbing curves (Fig. 2b). These results suggest a role for porin in the normal function of neurons in Drosophila since its reduced activity shortens lifespan and prematurely retards climbing ability.

The directed overexpression of the pro-survival $\mathrm{Bcl}-2$ homologue Buffy in these neurons resulted in increased lifespan and improved climbing ability. When Buffy is co-expressed with the porin-RNAi lines, the results 


\begin{tabular}{|c|c|c|}
\hline $\begin{array}{l}\text { Hsap } \\
\text { Dmel } \\
\text { Agam }\end{array}$ & 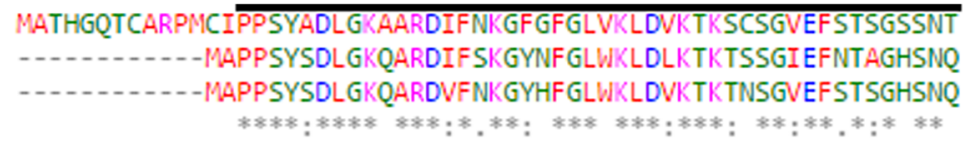 & $\begin{array}{l}60 \\
48 \\
48\end{array}$ \\
\hline $\begin{array}{l}\text { Hsap } \\
\text { Dmel } \\
\text { Agam }\end{array}$ & $\begin{array}{l}\text { DTGKVTGTLETKYKWCEYGLTFTEKWNTDNTLGTEIAIEDQICQGLKLTFDTTFSPNTGK } \\
\text { ESGKVFGSLETKYKVKDYGLTLTEKWNTDNTLFTEVAVQDdLLEGLKLSLEGNFAPQSGN } \\
\text { DTGKVFGSLETKYKVKEYGLNFSEKWNTDNTLTSEVSVENQLVKGLKVSFDGMFVPHTGS } \\
\begin{array}{l}:: * * * ;: * * * * * * \\
\text { Porin3_VDAC Domain }\end{array}\end{array}$ & $\begin{array}{l}120 \\
108 \\
108\end{array}$ \\
\hline $\begin{array}{l}\text { Hsap } \\
\text { Dmel } \\
\text { Agam }\end{array}$ & 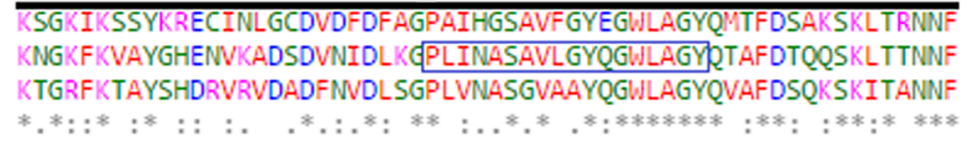 & $\begin{array}{l}180 \\
168 \\
168\end{array}$ \\
\hline $\begin{array}{l}\text { Hsap } \\
\text { Dmel } \\
\text { Agam }\end{array}$ & 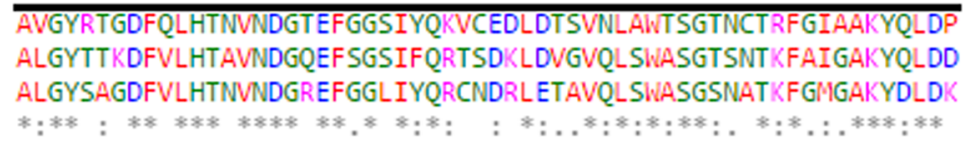 & $\begin{array}{l}240 \\
228 \\
228\end{array}$ \\
\hline $\begin{array}{l}\text { Fig. } 1 \text { Drosophila porin ha } \\
\text { the Porin domain is highly } \\
\text { domain, and a PDZ domain } \\
\text { resource search [30]. A Clust } \\
\text { NP_003366.2, Dmel is Dros } \\
\text { are identical, ":" indicate th } \\
\text { acids. Red is small hydrop }\end{array}$ & 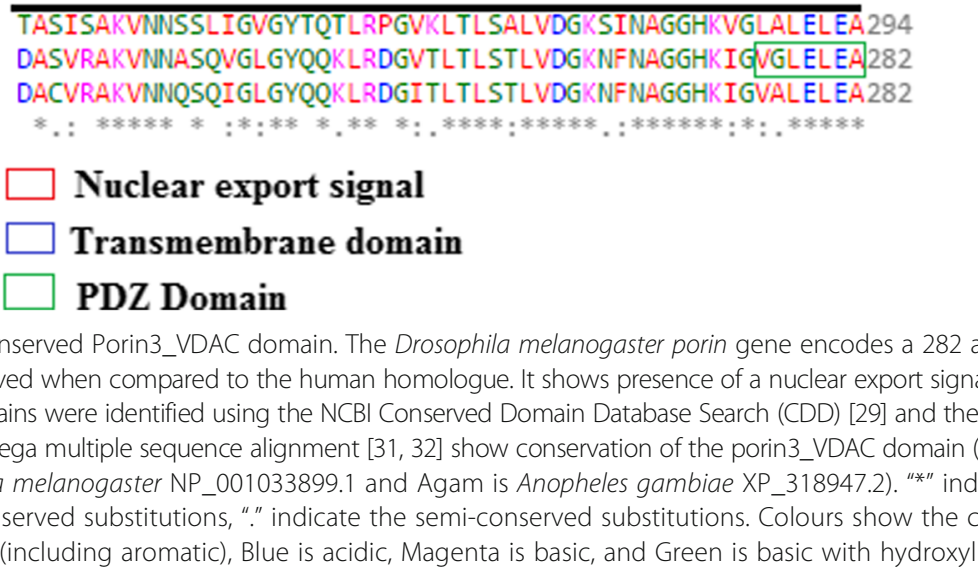 & $\begin{array}{l}\text { mino acids protein and } \\
\text { al (NES), a transmembrane } \\
\text { Eukaryotic Linear Motif } \\
\text { Hsap is Homo sapiens } \\
\text { icate the residues that } \\
\text { hemical nature of amino } \\
\text { or amine groups }\end{array}$ \\
\hline
\end{tabular}

indicate a median lifespan of 70 and 69 days when compared to 72 days for Buffy co-expression with lacZ control flies as determined by Log-rank test (Fig. 2c). The climbing ability of the porin-RNAi flies was significantly improved as determined by comparison of climbing curves of porin-RNAi flies at $95 \% \mathrm{CI}$ (Fig. 2b) with the flies that express porin-RNAi along with Buffy and with the control flies that co-expressed Buffy along with lacZ (Fig. 2d). Taken together these results suggest a prosurvival role for Buffy as observed by significant increases in the "healthspan" of porin-deficient flies.

\section{Inhibition of porin enhances $a$-synuclein-dependent phenotypes}

The expression of $\alpha$-synuclein in Ddc-Gal4-expressing neurons results in impaired locomotor function that has been attributed to cellular toxicity due to the accumulation of this protein. The co-expression of the porin$R N A i$ lines along with $\alpha$-synuclein, decreased survival and impaired climbing ability over time (Fig. 3). The median lifespan was 50 and 56 days for flies that expressed porin-RNAi along with $\alpha$-synuclein, compared to 60 days for control flies that co-expressed $\alpha$-synuclein along with lacZ, a significant decrease in survival for both RNAi lines (Fig. 3a) as determined by Log-rank (Mantel-Cox) test $(p<0.0001)$. A comparison of the climbing curves by nonlinear fitting at $95 \% \mathrm{CI}$ revealed they were significantly different (Fig. 3b), with CI of 0.04691 to 0.06795 and 0.030 to 0.050 for flies that expressed porin-RNAi along with $\alpha$-synuclein, compared to 0.06842 to 0.08366 for control flies that co-expressed $\alpha$-synuclein along with lacZ. This suggests that the inhibition of porin together with the expression of $\alpha$-synuclein in these neurons confers a significant health disadvantage, with marked decreases in survival and premature loss of climbing ability.

Inhibition of porin in the eye decreases ommatidia number and increases ommatidial disarray, phenotypes that are rescued by Buffy overexpression

When porin-RNAi is directed in the developing eye using the GMR-Gal4 transgene, it results in eyes with decreased number of ommatidia and higher disruption of the ommatidial array (Fig. 4ii, iii and $\mathrm{x}$ ) as determined 

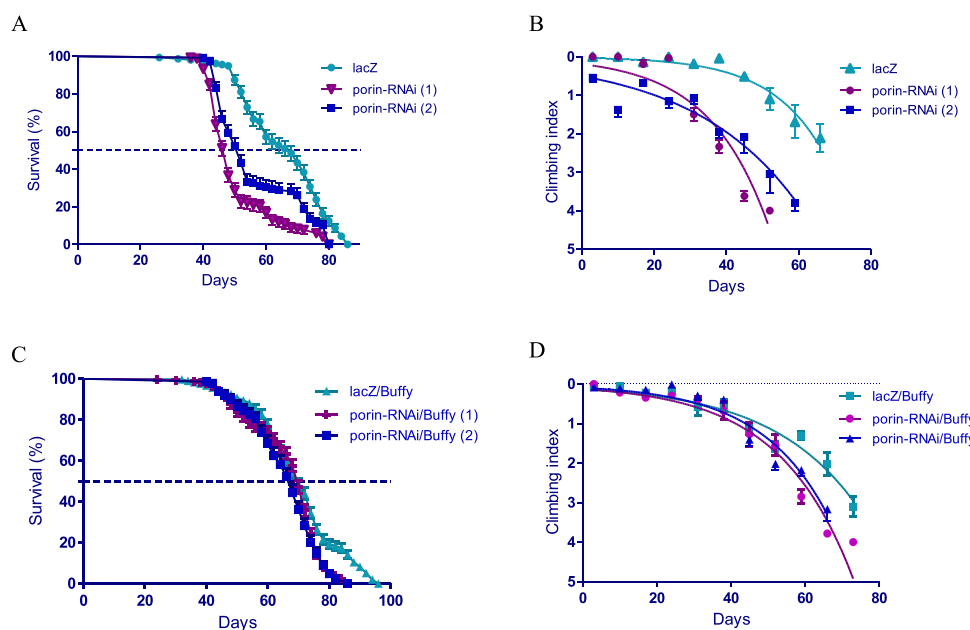

$\mathrm{D}$

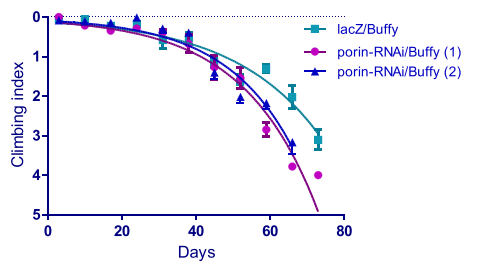

Fig. 2 Loss of porin activity decreases survival and impairs climbing ability. a The inhibition of porin in neurons using the Ddc-Gal4 transgene results in decreased median lifespan of 48 and 52 days when compared to 68 days for control flies that expresses UAS-lacZ. The genotypes are Ddc-Gal4/ UAS-lacZ, Ddc-Gal4/ UAS-porin-RNAi (1) and Ddc-Gal4/ UAS-porin-RNAi (2). Longevity is shown as percent survival ( $P<0.05$, determined by the log-rank (Mantel-Cox) test and $n \geq 200$ ). $\mathbf{b}$ The inhibition of porin in the Ddc-Gal4-expressing neurons resulted in a significant decrease in climbing ability as determined by nonlinear fitting of the climbing curves and comparing 95\% Cl. The genotypes are Ddc-Gal4/ UAS-lacZ, Ddc-Gal4/ UAS-porin-RNAi (1) and Ddc-Gal4/ UAS-porin-RNAi (2). Error bars indicate SEM and $n=50$. c The co-expression of Buffy with porin-RNAi result in the rescue of the observed phenotype of decreased survival, with a median survival of 70 and 69 days when compared to 72 days for controls. Genotypes are Ddc-Gal4 UAS-Buffy/ UAS-lacZ, Ddc-Gal4 UAS-Buffy/ UAS-porin-RNAi (1) and Ddc-Gal4 UAS-Buffy/ UAS-porin-RNAi (2). Longevity is shown as percent survival $(P<0.05$, determined by log-rank (Mantel-Cox) test with $n \leq 200$ ). $\mathbf{d}$ The inhibition of porin along with the overexpression of Buffy in the DA neurons results in the suppression of the age-dependent loss in climbing ability. The genotypes are Ddc-Gal4 UAS-Buffy/ UAS-lacZ, Ddc-Gal4 UAS-Buffy/ UAS-porin-RNAi (1) and Ddc-Gal4 UAS-Buffy/ UAS-porin-RNAi (2). Analysis was done by nonlinear fitting of the climbing curves and significance was determined by comparing the $95 \% \mathrm{Cl}$. Error bars indicate SEM and $n=50$

by a one-way analysis of variance followed by a Dunnett's multiple comparison test $p<0.0001$. Co-expression of porin with Buffy restored the mean number of ommatidia and the percentage disruption to control levels as determined by a one-way analysis of variance followed by a Dunnett's multiple comparison test $p>0.05$ (Fig. $4 \mathrm{v}$, vi and $\mathrm{xi}$ ). Taken together, these results suggest that porin may play a role in the development of the Drosophila eye and that Buffy suppresses the developmental eye defects that result from the inhibition of porin. The inhibition of porin along with $\alpha$-synuclein overexpression resulted in a significant decrease in the number of ommatidia due to fusion of ommatidia and an increase in the percentage disruption of the eye (Fig. 4 viii, ix and xii) as determined by a one-way analysis of variance followed by a Dunnett's multiple comparison test $p<0.0001$. This suggests an enhancement of the neurotoxic effects of the $\alpha$-synuclein-induced developmental eye defects in the presence of reduced porin activity.

\section{Discussion}

The multitude of functions attributed to mitochondrial porin or VDAC and its control of the entry and exit of mitochondrial metabolites makes it a key player in the cellular decisions that lead to either survival or death [1]. The expression of porin-RNAi in neurons under the direction of the $D d c$-Gal4 transgene results in shortened lifespan and a premature loss in locomotor ability, results that were consistent in both RNAi lines tested and that corroborate previous studies $[25,26]$. This gene product is involved in maintaining mitochondrial morphology, and its disruption leads to a host of phenotypes among them locomotor defects. In our study, we disrupted this protein in DA and other neurons, the results obtained signifies a close connection between porin and the progression of the PD-like phenotypes of shortened lifespan and an age-dependent loss in locomotor function. The comparison of climbing indices of flies at 40 days when most of them are alive to the control lines indicate a significant change in the phenotypes, these appears to be a strong indication of possible neurodegeneration.

The relationship between mitochondrial porin and PD susceptibility gene products has been investigated in other organisms $[12-14,49,50]$. The inhibition of porin along with the expression of $\alpha$-synuclein in Ddc-Gal4expressing neurons of Drosophila melanogaster results in the enhancement of the loss of $\alpha$-synuclein-induced phenotypes, with a decrease in lifespan and an impairment in climbing ability. Some studies have attributed the neurotoxicity of $\alpha$-synuclein to its interaction with electron transport chain components among them Complex I [51]. It has been suggested that $\alpha$-synuclein blocks the activity of porin and uses this channel to translocate 

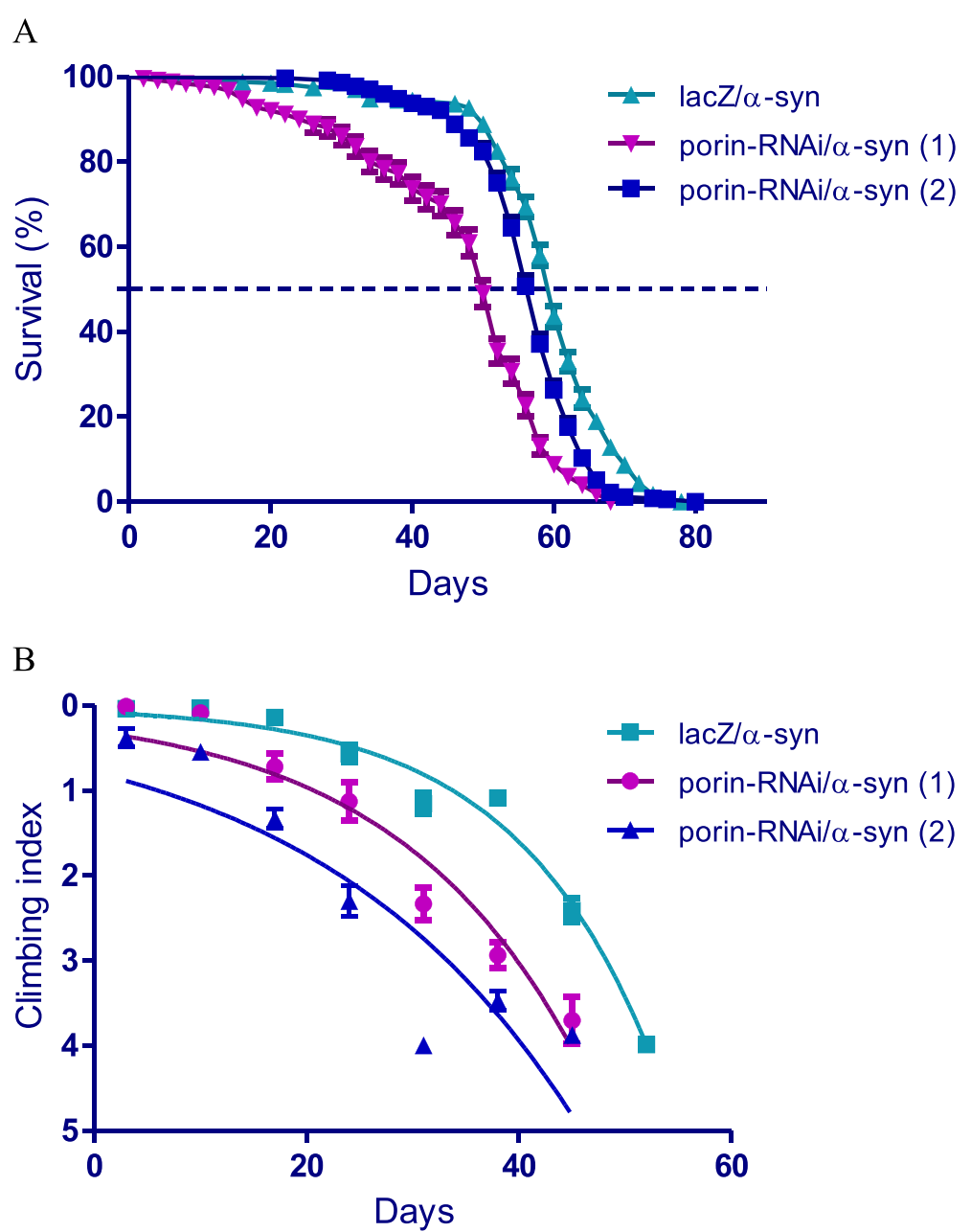

Fig. 3 Loss of porin function enhances the $a$-synuclein-induced reduction in lifespan and age-dependent loss of climbing ability. a The directed inhibition of porin along with a-synuclein expression in the neurons decreased lifespan with a median survival of 50 and 56 days when compared to 60 days for the control flies that express a-synuclein along with the lacZ transgene. Genotypes are UAS-a-synuclein; Ddc-Gal4/ UAS-lacZ, UAS-a-synuclein; Ddc-Gal4/UAS-porin-RNAi (1) and UAS-a-synuclein; Ddc-Gal4/ UAS-porin-RNAi (2). Longevity is shown as percent survival $(P<0.05$, determined by log-rank (Mantel-Cox) test with $n \leq 200)$. b The co-expression of porin-RNAi with $a$-synuclein resulted in reduction of climbing ability over time when compared to the controls. The genotypes are UAS-a-synuclein; Ddc-Gal4/UAS-lacZ, UAS-a-synuclein; Ddc-Gal4/UAS-porin-RNAi (1) and UAS-a-synuclein; Ddc-Gal4/UAS-porin-RNAi (2). Analysis was done by nonlinear fitting of the climbing curves and significance was determined by comparing the $95 \% \mathrm{Cl}$. Error bars indicate SEM and $n=50$

into the inner mitochondria [13] and that it preferentially interacts with mitochondrial membranes compared to other organelle membranes [52]. This association inhibits mitochondrial function and promotes reactive oxygen stress. Our study firstly inhibited the mitochondria porin and secondly expressed $\alpha$-synuclein in the same neurons, this resulted in the enhancement of the observed phenotypes, with shortened lifespan and severe reduction in climbing ability over time. It seems therefore that the combination effect of the directed inhibition of porin, and expression of $\alpha$-synuclein confers a greater disadvantage to "healthspan", albeit when altered in neurons. When altered individually, $\alpha$-synuclein-induced PD model, a well-studied and robust disease model in Drosophila $[16,22]$ result in shortened lifespan and impaired climbing ability. Inhibition of porin in the developing eye results in extensive ommatidial disruption and fewer ommatidia number, because of intensive fusion of the ommatidia with no distinct ommatidia detectable in most of the eyes analysed. We suggest that though $\alpha$-synuclein interacts with the mitochondria to result in disruption of mitochondria homeostasis, loss of porin in neurons seem to be independent of $\alpha$-synucleininduced phenotypes and this highlights the complexity of mechanisms involved in the pathogenesis of PD.

The association of porin with members of the $\mathrm{Bcl}-2$ family is well documented [7], and has been suggested to be a point of convergence for cell survival and death 

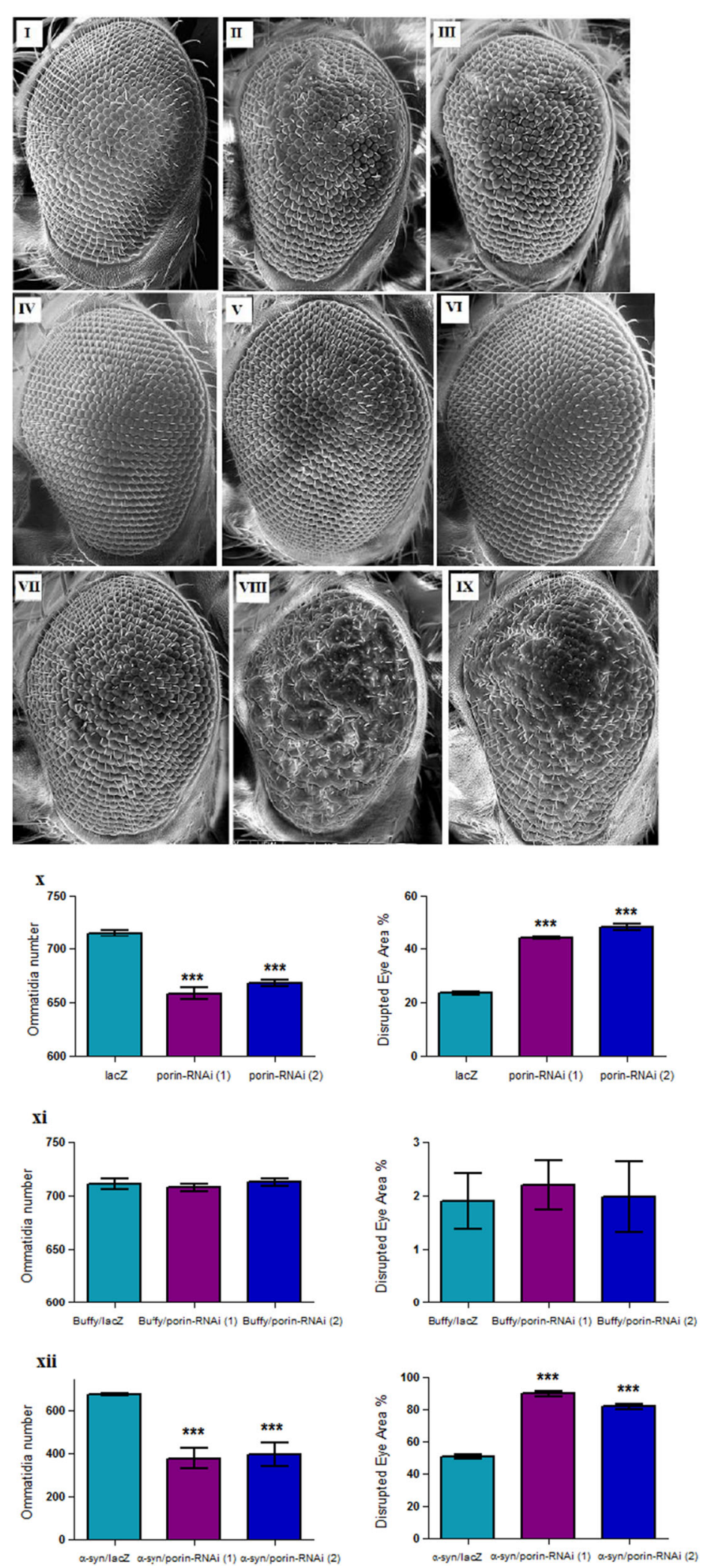

Fig. 4 (See legend on next page.) 
(See figure on previous page.)

Fig. 4 Inhibition of porin in the developing eye results in phenotypes that may be suppressed by Buffy and enhanced by a-synuclein. Scanning electron micrographs when porin is inhibited in the developing eye and co-expressed along with either Buffy or a-synuclein. The genotypes are (i) GMR-Gal4/ UAS-lacZ, (ii) GMR-Gal4/ UAS-porin-RNAi (1), (iii) GMR-Gal4/ UAS-porin-RNAi (2), (iv) UAS-Buffy; GMR-Gal4/ UAS-lacZ, (v) UAS-Buffy; GMR-Gal4/ UAS-porin-RNAi (1), (vi) UAS-Buffy; GMR-Gal4/UAS-porin-RNAi (2), (vii) UAS-a-synuclein; GMR-Gal4/UAS-lacZ, (viii) UAS-a-synuclein; GMR-Gal4/ UAS-porin-RNAi (1), and (ix) UAS-a-synuclein; GMR-Gal4/UAS-porin-RNAi (2). Biometric analysis when (x) porin is inhibited in the eye indicated decreased ommatidia number and higher percentage of ommatidial disruption when compared to the control. (xi) The overexpression of Buffy with porin-RNAi results in restoration of the number of ommatidia and the degree of ommatidial disruption to below the control levels. (xii) The inhibition of porin along with a-synuclein expression resulted in the enhancement of the eye phenotypes when compared to controls as displayed by the low number of ommatidia coupled by the high degree of disruption of the ommatidial array. All comparisons were determined by a one-way analysis of variance followed by a Dunnett's multiple comparison test $(P<0.05)$, error bars are SEM, asterisks $\left(^{*}\right)$ represent statistical significance and $n=10$

signals [3]. When we overexpressed Buffy, the sole prosurvival $\mathrm{Bcl}-2$ homologue, in Drosophila neurons, along with inhibition of porin via RNAi, the phenotypes associated with the loss-of-function of porin, shortened lifespan and impaired climbing ability, were suppressed. The survival-induced advantages of Buffy especially under conditions of stress are well documented [27, 28, $38,41,42]$, and so is the regulation of porin by $\mathrm{Bcl}-2$ proteins that underscores the importance of $\mathrm{Bcl}-2$ protein in life and death decisions. The overexpression of Buffy along with the inhibition of porin in Ddc-Gal4-expressing neurons and in the developing eye resulted in a suppression of the phenotypes. The excess Buffy product must therefore confer cellular advantages to the target cells and counteracts the toxic effects of porin inhibition, and demonstrates a wider role for the Drosophila prosurvival homologue, with potential involvement in the mitochondria-mediated cell death. The developmental expression patterns of Buffy and porin can shed light on the resulting phenotypes and possibly on the counteraction of the porin-induced phenotypes by overexpression of Buffy. One study has suggested that porin was not involved in debcl-induced cell death [25] and found that apoptosis induced by debcl overexpression was not inhibited by porin loss of function. As such it seems that the rescue of porin-induced phenotypes by Buffy are consistent with its action on the mitochondria directly or through other proteins in a dedicated pro-survival signalling pathway.

\section{Conclusions}

The inhibition of porin in the Ddc-Gal4-expressing neurons and the developing eye is rescued upon the overexpression of Buffy, a pro-survival Bcl-2 homologue. The co-expression of porin-RNAi along with $\alpha$-synuclein results in enhanced phenotypes, this highlights the complexity of $\alpha$-synuclein-induced mechanisms in the pathogenesis of PD, and in deed demonstrates the multi-faceted mechanisms involved in the aetiology of PD.

\section{Abbreviations}

Bcl-2: B cell lymphoma 2; Cl: Confidence interval; DA: Dopaminergic; Ddc: DOPA decarboxylase; GMR: Glass Multimer Reporter; RNAi: Ribonucleic acid interference; SEM: Standard error of the mean; VDAC: Voltagedependent anion channel

\section{Acknowledgements}

Not applicable.

\section{Funding}

PGM was been partially funded by Department of Biology Teaching Assistantships and a School of Graduate Studies Fellowship from Memorial University of Newfoundland. The research program of BES has been funded by the Natural Sciences and Engineering Research Council of Canada (NSERC) Discovery Grant. The funding bodies were not involved in the design of the study and in the collection, analysis, and interpretation of the data and writing the manuscript.

\section{Availability of data and material}

The datasets supporting the conclusions of this article are included within the article.

\section{Authors' contributions}

PGM performed the bioinformatic, survival, climbing/locomotion, biometric and statistical analyses. BES conceived and participated in the design, supervision of the study and revisions to the final draft of the manuscript. Both authors have read and approved the final manuscript.

\section{Competing interests}

The authors declare that they have no competing interests.

\section{Consent for publication}

Not applicable.

\section{Ethics approval}

This study has been conducted under the approval of the Animal Care Committee of Memorial University of Newfoundland as a Category of Invasiveness Level A protocol under the project title of "Genetic, biochemical and molecular analysis of cell survival and cell death in Drosophila melanogaster" (protocol number: 16-09-BS). Consent was not applicable for this study.

Received: 26 June 2016 Accepted: 15 November 2016

Published online: 24 November 2016

\section{References}

1. Shoshan-Barmatz V, De Pinto V, Zweckstetter M, Raviv Z, Keinan N, Arbel N. VDAC, a multi-functional mitochondrial protein regulating cell life and death. Mol Aspects Med. 2010;31:227-85.

2. Ryerse J, Blachly-Dyson E, Forte M, Nagel B. Cloning and molecular characterization of a voltage-dependent anion-selective channel (VDAC) from Drosophila melanogaster. Biochim Biophys Acta. 1997;1327:204-12.

3. Shoshan-Barmatz V, Ben-Hail D. VDAC, a multi-functional mitochondrial protein as a pharmacological target. Mitochondrion. 2012;12:24-34. 
4. Craigen WJ, Graham BH. Genetic strategies for dissecting mammalian and Drosophila voltage-dependent anion channel functions. J Bioenerg Biomembr. 2008:40:207-12.

5. De Stefani D, Bononi A, Romagnoli A, Messina A, De Pinto V, Pinton P, et al. VDAC1 selectively transfers apoptotic Ca2+ signals to mitochondria. Cell Death Differ. 2012;19:267-73.

6. Pastorino JG, Hoek JB. Regulation of hexokinase binding to VDAC. J Bioenerg Biomembr. 2008;40:171-82.

7. Tsujimoto Y, Shimizu S. VDAC regulation by the Bcl-2 family of proteins. Cell Death Differ. 2000;7:1174-81.

8. Cheng EH, Sheiko TV, Fisher JK, Craigen WJ, Korsmeyer SJ. VDAC2 inhibits BAK activation and mitochondrial apoptosis. Science (New York, NY). 2003; 301:513-7.

9. Ramírez CM, González M, Díaz M, Alonso R, Ferrer I, Santpere G, et al. VDAC and ER interaction in caveolae from human cortex is altered in Alzheimer's disease. Mol Cell Neurosci. 2009;42:172-83.

10. Yoo BC, Fountoulakis M, Cairns N, Lubec G. Changes of voltage dependent anion selective channel proteins VDAC1 and VDAC2 brain levels in patients with Alzheimer's disease and Down Syndrome. Electrophoresis. 2001. doi:10, 1002/1522-2683(200101)22:1<172::AID-ELPS172>3.0.CO;2-P.

11. Premkumar A, Simantov R. Mitochondrial voltage-dependent anion channel is involved in dopamine-induced apoptosis. J Neurochem. 2002;82:345-52.

12. Sun Y, Vashisht AA, Tchieu J, Wohlschlegel JA, Dreier L. Voltage-dependent anion channels (VDACs) recruit Parkin to defective mitochondria to promote mitochondrial autophagy. J Biol Chem. 2012;287:40652-60.

13. Rostovtseva TK, Gurnev PA, Protchenko O, Hoogerheide DP, Yap TL, Philpott CC, et al. a-Synuclein Shows High Affinity Interaction with Voltage-dependent Anion Channel, Suggesting Mechanisms of Mitochondrial Regulation and Toxicity in Parkinson Disease. J Biol Chem. 2015;290:18467-77.

14. Chu Y, Goldman JG, Kelly L, He Y, Waliczek T, Kordower JH. Abnormal alphasynuclein reduces nigral voltage-dependent anion channel 1 in sporadic and experimental Parkinson's disease. Neurobiol Dis. 2014;69:1-14.

15. Shen J, Du T, Wang X, Duan C, Gao G, Zhang J, et al. a-Synuclein amino terminus regulates mitochondrial membrane permeability. Brain Res. 2014; 1591:14-26

16. Feany MB, Bender WW. A Drosophila model of Parkinson's disease. Nature. 2000;404:394-8.

17. Auluck PK, Chan HY, Trojanowski JQ, Lee VM, Bonini NM. Chaperone suppression of alpha-synuclein toxicity in a Drosophila model for Parkinson's disease. Science. 2002:295:865-8.

18. Buttner S, Broeskamp F, Sommer C, Markaki M, Habernig L, AlavianGhavanini A, et al. Spermidine protects against alpha-synuclein neurotoxicity. Cell Cycle (Georgetown, Tex). 2014;13:3903-8.

19. Kong Y, Liang X, Liu L, Zhang D, Wan C, Gan Z, et al. High Throughput Sequencing Identifies MicroRNAs Mediating alpha-Synuclein Toxicity by Targeting Neuroactive-Ligand Receptor Interaction Pathway in Early Stage of Drosophila Parkinson's Disease Model. PLoS One. 2015;10:e0137432.

20. Wang B, Liu Q, Shan H, Xia C, Liu Z. Nrf2 inducer and cncC overexpression attenuates neurodegeneration due to alpha-synuclein in Drosophila. Biochem Cell Biol. 2015;93:351-8.

21. Zhu ZJ, Wu KC, Yung WH, Qian ZM, Ke Y. Differential interaction between iron and mutant alpha-synuclein causes distinctive Parkinsonian phenotypes in Drosophila. Biochim Biophys Acta. 1862;2016:518-25.

22. Staveley BE. Drosophila Models of Parkinson Disease. In: Movement Disorders: Genetics and Models. Second edn. Edited by LeDoux MS. London: Elsevier Science; 2015. 345-354.

23. Botella JAA, Bayersdorfer F, Gmeiner F, Schneuwly S. Modelling Parkinson's disease in Drosophila. Neuromolecular Med. 2009;11:268-80.

24. Brand $\mathrm{AH}$, Perrimon N. Targeted gene expression as a means of altering cell fates and generating dominant phenotypes. Development. 1993;1 18:401-15.

25. Park J, Kim Y, Choi S, Koh H, Lee S-HH, Kim J-MM et al. Drosophila Porin VDAC affects mitochondrial morphology. PloS ONE. 2010;5(10):e13151.

26. Graham BH, Li Z, Alesii EP, Versteken P, Lee C, Wang J, et al. Neurologic dysfunction and male infertility in Drosophila porin mutants: a new model for mitochondrial dysfunction and disease. J Biol Chem. 2010;285:11143-53.

27. M'Angale PG, Staveley BE. The BCl-2 homologue Buffy rescues alphasynuclein-induced Parkinson disease-like phenotypes in Drosophila. BMC Neurosci. 2016;17:24

28. M'Angale PG, Staveley BE. The HtrA2 Drosophila model of Parkinson Disease is suppressed by the pro-survival BCl-2 Buffy. Genome 2016; In Press.
29. Marchler-Bauer A, Derbyshire MK, Gonzales NR, Lu S, Chitsaz F, Geer LY, et al. CDD: NCBI's conserved domain database. Nucleic Acids Res. 2015:43:D222-6.

30. Dinkel H, Van Roey K, Michael S, Davey NE, Weatheritt RJ, Born D, et al. The eukaryotic linear motif resource ELM: 10 years and counting. Nucleic Acids Res. 2013. doi:10.1093/nar/gkt1047.

31. Sievers F, Wilm A, Dineen D, Gibson TJ, Karplus K, Li W, et al. Fast, scalable generation of high-quality protein multiple sequence alignments using Clustal Omega. Mol Syst Biol. 2011;7:539.

32. Goujon M, McWilliam H, Li W, Valentin F, Squizzato S, Paern J, et al. A new bioinformatics analysis tools framework at EMBL-EBI. Nucleic Acids Res. 2010;38:W695-9.

33. la Cour T, Kiemer L, Mølgaard A, Gupta R, Skriver K, Brunak S. Analysis and prediction of leucine-rich nuclear export signals. Protein Eng Des Sel. 2004; 17:527-36.

34. Fisher B, Weiszmann R, Frise $\mathrm{E}$, Hammonds A, Tomancak $\mathrm{P}$, Beaton A et al. BDGP insitu homepage. In.; 2012.

35. Oliva M, De Pinto V, Barsanti P, Caggese C. A genetic analysis of the porin gene encoding a voltage-dependent anion channel protein in Drosophila melanogaster. Mol Genet Genomics. 2002;267:746-56.

36. Freeman M. Reiterative use of the EGF receptor triggers differentiation of all cell types in the Drosophila eye. Cell. 1996;87:651-60.

37. Li H, Chaney S, Roberts IJ, Forte M, Hirsh J. Ectopic G-protein expression in dopamine and serotonin neurons blocks cocaine sensitization in Drosophila melanogaster. Curr Biol. 2000;10:211-4.

38. Quinn L, Coombe M, Mills K, Daish T, Colussi P, Kumar S, et al. Buffy, a Drosophila Bcl-2 protein, has anti-apoptotic and cell cycle inhibitory functions. EMBO J. 2003;22:3568-79.

39. Brachmann CB, Jassim OW, Wachsmuth BD, Cagan RL. The Drosophila bcl-2 family member dBorg-1 functions in the apoptotic response to UVirradiation. Curr Biol. 2000;10:547-50.

40. Colussi PA, Quinn LM, Huang DC, Coombe M, Read SH, Richardson H, et al. Debcl, a proapoptotic $\mathrm{BCl}-2$ homologue, is a component of the Drosophila melanogaster cell death machinery. J Cell Biol. 2000;148:703-14.

41. M'Angale PG, Staveley BE. BCl-2 homologue Debcl enhances a-synucleininduced phenotypes in Drosophila. Peer J. 2016;4:e2461.

42. M'Angale PG, Staveley BE. Inhibition of Atg6 and Pi3K59F autophagy genes in neurons decreases lifespan and locomotor ability in Drosophila melanogaster. Gen Mol Res. 2016; 15:gmr15048953.

43. Todd AM, Staveley BE. Expression of Pink1 with alpha-synuclein in the dopaminergic neurons of Drosophila leads to increases in both lifespan and healthspan. Genet Mol Res. 2012;11:1497-502.

44. Staveley BE, Phillips JP, Hilliker AJ. Phenotypic consequences of copper-zinc superoxide dismutase overexpression in Drosophila melanogaster. Genome. 1990;33:867-72.

45. Todd AM, Staveley BE. Novel assay and analysis for measuring climbing ability in Drosophila. Dros Info Serv. 2004;87:101-7.

46. Schneider CA, Rasband WS, Eliceiri KW. NIH Image to ImageJ: 25 years of image analysis. Nat Methods. 2012;9:671-5.

47. M'Angale PG, Staveley BE. Effects of a-synuclein expression in the developing Drosophila eye. Dros Info Serv. 2012;95:85-9.

48. Dinkel H, Van Roey K, Michael S, Kumar M, Uyar B, Altenberg B, et al. ELM 2016-data update and new functionality of the eukaryotic linear motif resource. Nucleic Acids Res. 2016:44:D294-300.

49. Geisler S, Holmstrom KM, Skujat D, Fiesel FC, Rothfuss OC, Kahle PJ, et al. PINK1/Parkin-mediated mitophagy is dependent on VDAC1 and p62/ SQSTM1. Nat Cell Biol. 2010;12:119-31.

50. Narendra D, Kane LA, Hauser DN, Fearnley IM, Youle RJ. p62/SQSTM1 is required for Parkin-induced mitochondrial clustering but not mitophagy; VDAC1 is dispensable for both. Autophagy. 2010:6:1090-106.

51. Chinta SJ, Mallajosyula JK, Rane A, Andersen JK. Mitochondrial alpha-synuclein accumulation impairs complex I function in dopaminergic neurons and results in increased mitophagy in vivo. Neurosci Lett. 2010;486:235-9.

52. Nakamura K, Nemani VM, Wallender EK, Kaehlcke K, Ott M, Edwards RH. Optical reporters for the conformation of alpha-synuclein reveal a specific interaction with mitochondria. J Neurosci. 2008:28:12305-17. 